\title{
CONTRIBUIÇÃO AO CONHECIMENTO DE STRONGYLURIS OSCARI TRAVASSOS, 1923 (NEMATODA, SUBULUROIDEA) ${ }^{1}$
}

\author{
ANNA KOHN, * R. MAGALhães PINTO ** \\ e \\ BERENICE M. M. FERNANDES ** \\ Instituto Oswaldo Cruz, Rio de Janeiro, Guanabara \\ (Com 9 figuras e 1 quadro)
}

Sumário: $\mathrm{Na}$ presente nota é feita uma revisão da espécie Strongyluris oscari Travassos, 1923, baseada no estudo dos exemplares tipo e de material proveniente do Ceará, retificando-se a descrição original e propondo Strongyluris sci Travassos, 1926 como seu sinônimo.

$\mathrm{D}$ E material proveniente do Ceará, tivemos oportunidade de estudar os helmintos parasitos de lagarto (Tropidurus torquatus) (Wied) gentilmente cedidos pela Prof. ${ }^{\text {a }}$ Maria Auxiliadora de Souza, pertencentes ao gênero Strongyluris Mueller, 1894.

\section{MATERIAL E MÉTODOS}

Nossos estudos foram baseados no ma. terial tipo de Strongyluris oscari Travassos, 1923, e de Strongyluris sai Travassos, 1926, bem como em 8 exemplares provenientes do Ceará.

Damos a seguir a relação do materia! tipo estudado com as respectivas anotações originais, da Coleção Helmintológica do I.O.C.
N. ${ }^{\circ} 4.100$ - Strongyluris oscari Travassos, 1923; Tropidurus; intestino grosso; S. João - Mato Grosso; Travassos col. 8/VII/922; Travassos det. I/923; autópsia n. ${ }^{\circ}$ 2.445. Obs. - Passou para bálsamo sob o n. $^{\circ} 30.836$.

N. 4.101 - Strongyluris oscari Travassos, 1923; Tropidurus; intestino grosso; S. João - Mato Grosso; Travassos col. 22/VIJ/922; Travassos det. I/923; autópsia n. ${ }^{\circ} 2.624$.

N. ${ }^{\circ} 4.102$ - Strongyluris oscari Travassos, 1923; Tropidurus; intestino grosso; S. João - Mato Grosso; Travassos col. 24/VII/922; Travassos det. I/923; autópsia n. ${ }^{\circ}$ 2.636. Obs. - Passou para bálsamo sob n. ${ }^{\circ} 30.837 \mathrm{a}-\mathrm{d}$.

N. ${ }^{\circ} 4.103$ - Strongyluris oscari Travassos, 1923; Tropidurus; cavidade auricular? (naturalmente o exemplar foi conduzido por algum instrumento);

1 Entregue para publicação a 18 de abril de 1973.

Trabalho do Laboratório de Helmintologia do Departamento de Zoologia

Médica do Instituto Oswaldo Cruz, realizado com auxílio do CNPq.

* Pesquisadora do Instituto Oswaldo Cruz e bolsista do CNPq.

** Bolsistas do CNPq. 
S. João - Mato Grosso; Travassos col. 24/VII/922; Travassos det. I/923; autópsia n. ${ }^{\circ}$ 2.636. Obs. - Material em bálsamo sob o n." 30.838a-c.

N." 4.104 - Strongyluris osccri Travassos, 1923; Tropidurus; intestino grosso; S. João - Mato Grosso; Travassos col. 24/VII/922; Travassos det. I/923; autópsia n. ${ }^{\circ}$ 2.637. Obs. - Material em bálsamo sob o n. ${ }^{\circ} 30.839$ a-d. Neótipo macho n. ${ }^{\circ} 30.839 \mathrm{c}$; neótipo fêmea n. ${ }^{\circ} 30.839$ a e parátipos $n .^{\circ} \mathrm{s}$ $30.839 \mathrm{~b}$ e d.

N. ${ }^{\circ} 4.105$ - Strongyluris oscari Travassos, 1923; Tropidurus; intestino grosso; S. João - Mato Grosso; Travassos col. 24/VII/922; Travassos det. I/923; autópsia n. 2.638. Obs. Vidro vazio (Material reestudado por Alho em 1969).

N. 4.904 - Strongyluris sai Travassos, 1926; Camaleão verde; intestino grosso; Angra dos Reis, Estado do Rio; Travassos col. 2/I/925; Travassos det.; autópsia n. ${ }^{\circ}$ 3.319. Obs. Passou para bálsamo sob o n. ${ }^{\circ} 30.840 a-b$.

N. 4.905 - Strongyluris sai Travassos, 1926; intestino grosso; Angra dos Reis, Estado do Rio; Travassos col. 29/I/1925; Travassos det.; autópsia n. ${ }^{\circ} 30.841 \mathrm{a}-\mathrm{e}$.

N. ${ }^{\circ} 30.842 \mathrm{a}-\mathrm{h}$ - Strongyluris oscari 'Travassos, 1923; intestino grosso; Fortaleza - Ceará; M. A. Souza Col. XI/972; Kohn, Pinto \& Fernandes det. I/973.

Os helmintos estavam conservados na Coleção Helmintológica do Instituto Oswaldo Cruz, em formol a $5 \%$ ou no líquido de Railliet \& Henry e o material proveniente da autópsia do Ceará foi coletado em soro fisiológico e fixado a quente no líquido de Railliet \& Henry. Foi corado em carmim acético, diafanizado com fenol e creosoto de faia e montado definitivamente em bálsamo do Canadá.

\section{RESULTADOS E DISCUSSÃO}

Em 1923 Travassos no trabalho "Informações sobre a fauna helmintológica de Mato Grosso" propõe Stron. gyluris oscari, primeira espécie do gênero encontrada no Brasil, parasito de intestino grosso de Tropidurus sp., dando uma breve descrição. Posteriormente em 1926, o mesmo autor descreve e representa Strongyluris sai, parasito de intestino grosso de camaleão verde, de Angra dos Reis, Estado do Rio de Janeiro.

Em 1935, Pereira identifica e descreve nematódeos parasitas de Tropidurus provenientes da Paraíba a $S$. os. cari Travassos, 1923.

Alho, $€ m 1969$ propõe mais duas espécies para o gênero: $S$. travassosi e $S$. freitasi, redescreve $S$. oscari do material tipo e repete a descrição de Travassos para $S$. sai.

Aproveitamos a oportunidade para rever o material tipo de $S$. oscari e $S$. sai depositado em nossas coleções $\mathrm{e}$ constatamos após estudos detalhados, que $S$. oscari Travassos, 1923 apresenta 10 pares de papilas na cauda dos machos ao invés dos 8 pares originalmente descritos, sendo idêntica a $S$. sai. Não encontramos nenhum caráter que permitisse a separação dessas duas espécies. Portanto, permanece $S$. oscari Travassos, 1923, por ordem prioritária, ficando $S$. sai como seu sinônimo. Não analisamos a validade das outras espécies brasileiras: $S$. freitasi Alho, 1969, e S. travassosi Alho, 1969, pois parte do material tipo depositado em nossas coleções não se encontra em boas condições, o que nos dificultou um diagnóstico preciso, bem como não tivemos acesso ao material estudado por Pereira em 1935.

Não tendo Travassos em 1923 escolhido o tipo de $S$. oscari, Alho, em 1969 designou o exemplar n. ${ }^{\circ} 4.105$ da Coleção Helmintológica do Instituto 


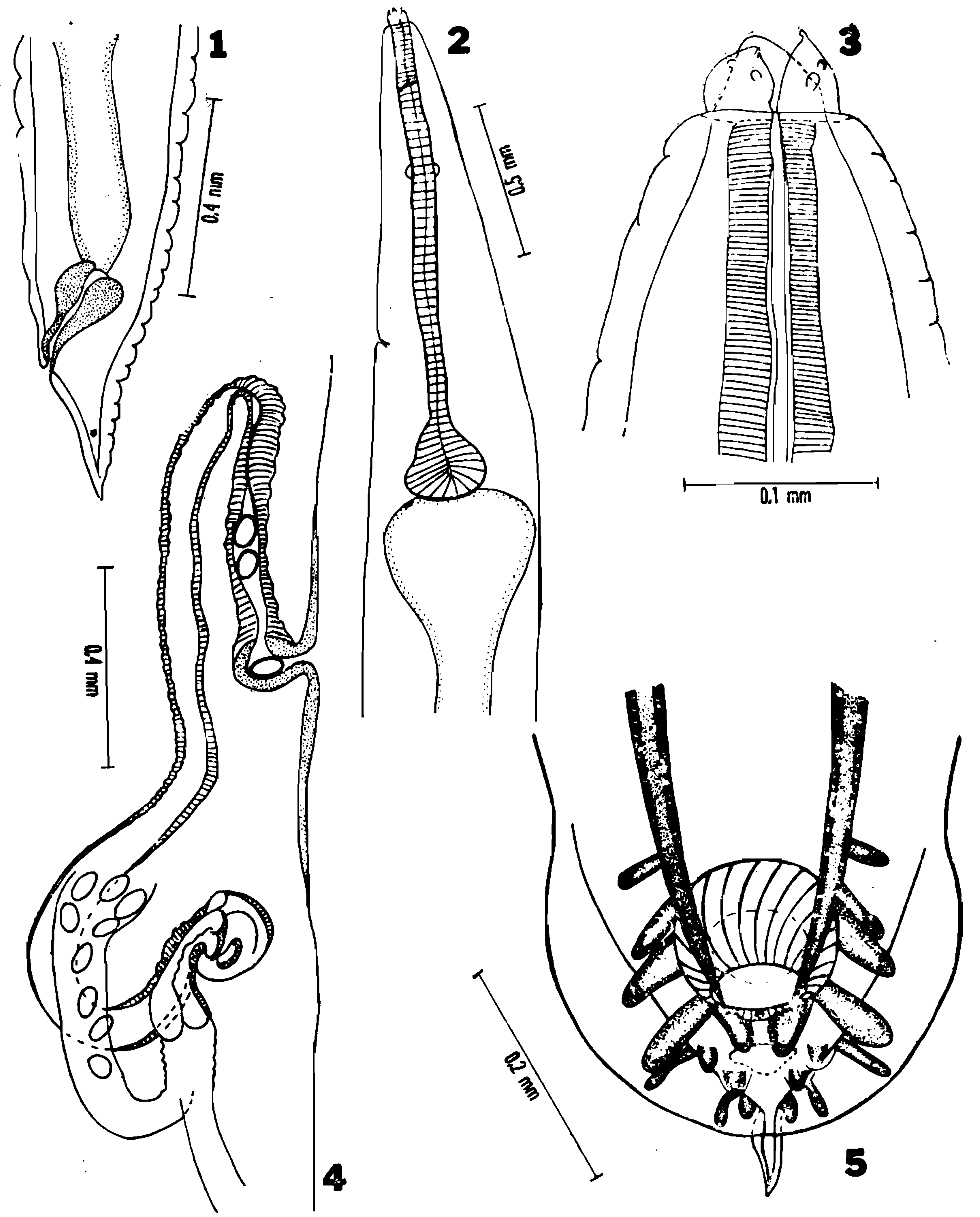

Strongyluris oscari Travassos, 1923; Fig. 1 - Extremidade posterior da fêmea (Col. Helm. I.O.C. n. ${ }^{\circ} 30.842-d$ ); Fig. 2 - Extremidade anterior da fêmea (Col. Helm. I.O.C. n. ${ }^{\circ} 30.842-\mathrm{g}$ ); Fig. 3 - Extremidade anterior do macho (Col. Helm. I.O.C. n. ${ }^{\circ}$ 30.842-b); Fig. 4 - Ovejetor (Col. Helm. I.O.C. n. ${ }^{\circ}$ 30.842-f); Fig. 5 - Extremidade posterior do macho (Col. Helm. I.O.C. n. $\left.{ }^{\circ} 30.842-c\right)$. Figuras originais. 
Oswaldo Cruz para tal finalidade. Por ocasião de nossos estudos, ao revermos todo o material disponível do gê-. nero, não conseguimos encontrar o exemplar n. 4.105 referido por Alho como tendo sido depositado em nossas coleções. (Cf.: Rev. Brasil. Biol. 1969, 29 (1): 72). Desta forma, resolvemos designar como neótipo macho o exemplar $\mathrm{n}^{\circ} 30.839 \mathrm{c}$, como neótipo fêmea o exemplar n. ${ }^{\circ} 30.839$ a e como parátipos os exemplares n.os $30.839 \mathrm{~b}-\mathrm{d}$.

Damos a seguir, a descrição e figuras dos exemplares provenientes do Ceará, identificados a $S$. oscari, Travassos, 1923, e também medidas e figuras originais do material tipo.

\section{Strongyluris oscari Travassos, 1923}

Sinonímia e referências:

Strongyluris oscari: Travassos, 1923: 6; Strongyluris oscari: Travassos, 1926: 3 , Strongyluris sai: Travassos, 1926: 3-4, figs. 1-5;

Strongyluris oscari: Pereira, 1935: 25;

Strongyluris sai: Caballero, 1938: 229;

Strongyluris oscari: Yamaguti, 1961: 152:

Strongyluris sai: Yamaguti, 1961: 152;

Strongyluris oscari: Caballero, 1968: 178-179;

Strongyluris oscari: Alho, 1969: 67, 68,

71, 72, 73, figs. 9-11;

Strongyluris sai: Alho, 1969: 71, 72, 73.

\section{DESCRIÇÃO:}

Comprimento - Machos: 8,23 a $8,80 \mathrm{~mm}$ Fêmeas: 8,38 a $9,02 \mathrm{mr}$

Largura - Machos: 0,53 a $0,56 \mathrm{~mm}$ Fêmeas: $0,60 \mathrm{~mm}$

Corpo fusiforme. Cutícula estriada trans. versalmente. Boca com 3 lábios, tendo cada um, 2 papilas laterais. Os lábios medem 0,035 a $0,038 \mathrm{~mm}$ de comprimento por 0,031 a $0,035 \mathrm{~mm}$ de largura nos machos e 0,040 a $0,045 \mathrm{~mm}$ de comprimento por
0,033 a $0,042 \mathrm{~mm}$ de largura nas fêmeas. Faringe relativamente longa, apresentando posteriormente uma curvatura de modo a formar ângulo no ponto de união com o esôfago; mede 0,17 a $0,21 \mathrm{~mm}$ de comprimento por 0,04 a $0,05 \mathrm{~mm}$ de largura nos machos e 0,18 a $0,23 \mathrm{~mm}$ de comprimento por 0,05 a $0,07 \mathrm{~mm}$ de largura nas fêmeas. Esôfago longo e delgado, medindo, sem o bulbo, 1,10 a $1,20 \mathrm{~mm}$ de comprimento por 0,06 a $0,08 \mathrm{~mm}$ de largura nos machos e 1,10 a $1,30 \mathrm{~mm}$ de comprimento por 0,08 a $0,09 \mathrm{~mm}$ de largura nas fêmeas. Bulbo esofagiano, com válvulas quitinosas, mede 0,27 a $0,28 \mathrm{~mm}$ de comprimento por 0,20 a $0,26 \mathrm{~mm}$ de largura nos machos e 0,19 a $0,31 \mathrm{~mm}$ de comprimento por 0,19 a $0,30 \mathrm{~mm}$ de largura nas fêmeas. Intestino retilíneo, com a extremidade anterior dilatada. Poro excretor situado cerca de $0,91 \mathrm{~mm}$ da extremidade anterior dos machos e 0,89 a $1,06 \mathrm{~mm}$ da extremidade anterior das fêmeas. Anel nervoso distando $0,59 \mathrm{~mm} \mathrm{da}$ extremidade anterior dos machos e $0,50 \mathrm{~mm}$ da extremidade anterior das fêmeas.

Fêmeas ovíparas, didelfas, anfidelfas, com vulva submediana, de lábios salientes, situada a 2,82 a $3,20 \mathrm{~mm}$ da extremidade posterior. Ovos com 0,060 a $0,071 \mathrm{~mm}$ de comprimento por 0,041 a $0,045 \mathrm{~mm}$ de largura. Reto com 0,23 a $0,24 \mathrm{~mm}$ de comprimento. Ânus distando 0,27 a $0,30 \mathrm{~mm}$ da extremidade posterior. Cauda cônica, apresentando um par de papilas (Fig. 1).

Machos com cauda truncada e asas caudais estreitas, apresentando 10 pares de papilas pedunculadas. Ventosa de rebordos fortes, circular, situada logo acima do ânus, com 0,11 a $0,14 \mathrm{~mm}$ de comprimento por 0,13 a $0,14 \mathrm{~mm}$ de largura, com uma peciuena papila no rebordo posterior (Fig. N. ${ }^{\circ}$ 5). Ânus distando de 0,04 a $0,08 \mathrm{~mm}$ da extremidade caudal, que apresenta um espinho terminal de cerca de 0,038 a $0,047 \mathrm{~mm}$ de comprimento. Espículos subiguais, medindo 0,81 a $0,98 \mathrm{~mm}$ de comprimento.

Habitat - Intestino grosso de Tropidurus torquatus (Wied).

Distribuição geográfica: Fortaleza, Estado do Ceará, Brasil.

Material estudado e depositado na Coleção Helmintológica do Instituto Oswaldo Cruz sob o N. 30.842 a-h. 


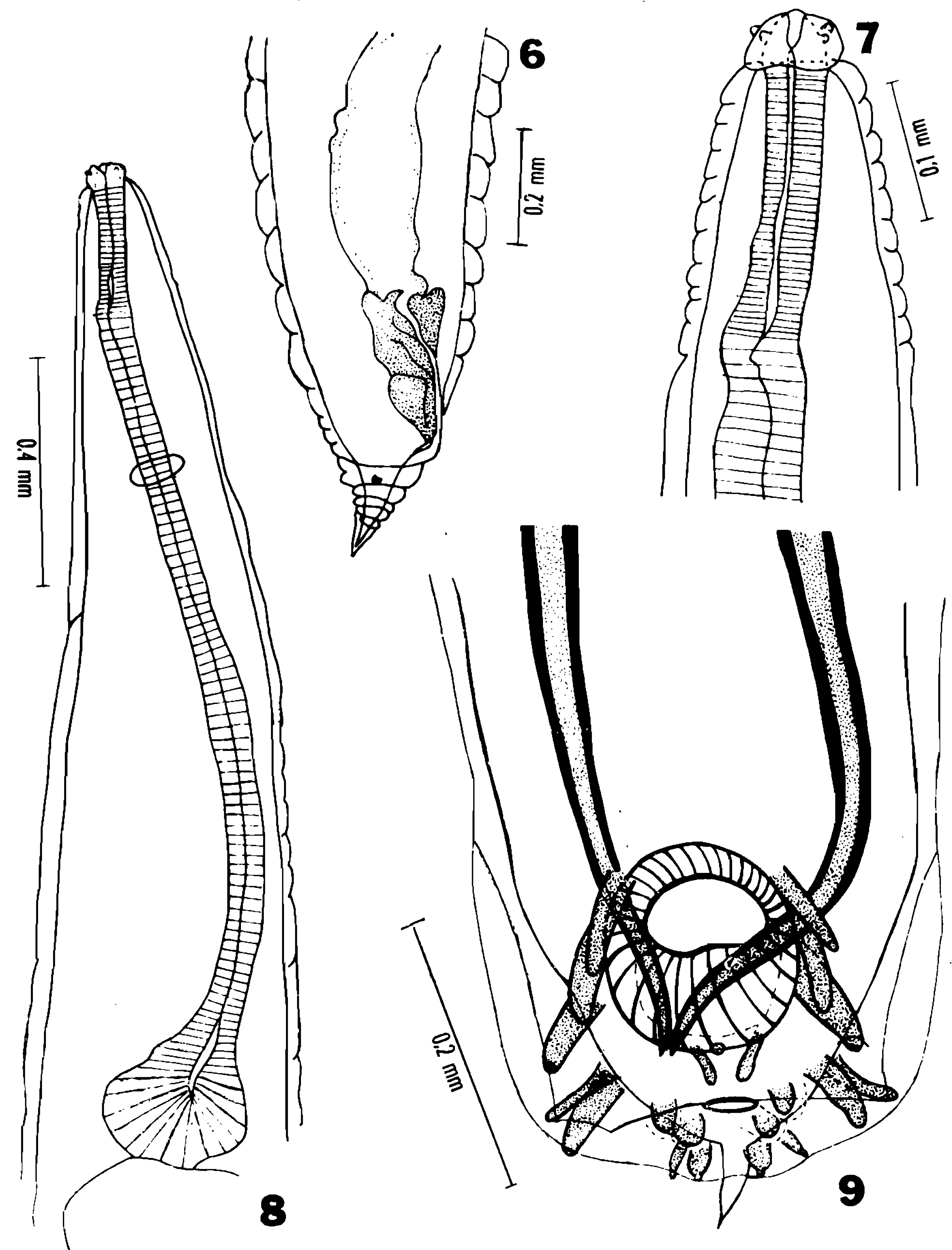

Strongyluris oscari Travassos, 1923: Fig. 6 - Extremidade posterior do neótipo fêmea (Col. Helm. I.O.C. n. ${ }^{\circ} 30.839-a$ ); Fig. 7 - Extremidade anterior do neótipo macho (Col. Helm. I.O.C. n. ${ }^{\circ} 30.839-\mathrm{c}$ ); Fig. 8 - Extremidade anterior do neótipo macho (Col. Helm. I.O.C. n. ${ }^{\circ} 30.839-\mathrm{c}$ ); Fig. 9 - Extremidade posterior do neótipo macho (Col. Helm. I.O.C. n. ${ }^{\circ}$ 30.839-c). Figuras originais. 
Q U A D R O I

QUADRO COMPARATIVO ENTRE O MATERIAL TIPO DE S. OSCARI TRAV., 1923, S. SAI TRAV., 1926 E O MATERIAL PROVENIENTE DO CEARÁ. (TODAS AS MEDIDAS EM MM.)

\begin{tabular}{|c|c|c|c|c|c|c|c|c|}
\hline \multirow[t]{2}{*}{ Espécies } & \multicolumn{2}{|c|}{$\begin{array}{l}\text { S. osrari Trav., } 1923 \\
\text { medidas originais }\end{array}$} & \multicolumn{2}{|c|}{$\begin{array}{l}\text { S. oscari Trav., } 1923 \\
\text { med. orig. do material tipo }\end{array}$} & \multicolumn{2}{|c|}{$\begin{array}{l}\text { S. oscari Trav., } 1923 \\
\text { medidas seg. Trav., } 1923\end{array}$} & \multicolumn{2}{|c|}{$\begin{array}{c}\text { S. sai Trav., } 1926 \\
\text { medidas seg. Trav., } 1926\end{array}$} \\
\hline & macho & fêmea & macho & fêmea & macho & fêmea & macho & fêmea \\
\hline Comprimento & $8,23-8,80$ & $8,38-9,02$ & $8,42-11,6$ & $9,26-10,23$ & 10 & 10 & 11 & $12-14$ \\
\hline Largura & $0,53-0,56$ & 0,60 & $0,53-0,60$ & $0,32-0,64$ & 0,5 & 0,5 & 0,43 & 0,75 \\
\hline Faringe & $\begin{array}{l}0,17-0,21 \\
0,04 \div 0,05\end{array}$ & $\begin{array}{l}0,18-0,23 \\
0,05-0,07\end{array}$ & $\begin{array}{l}0,21-0,22 \\
0,05-0,06\end{array}$ & $\begin{array}{l}0,23-0,24 \\
0,06-0,08\end{array}$ & $\begin{array}{l}0.2 \\
\times \\
0,5\end{array}$ & & 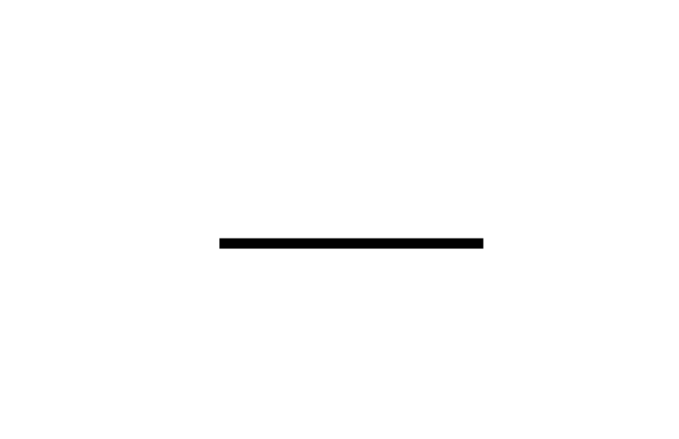 & - \\
\hline Esôfago & $\begin{array}{l}1,10-1,20 \\
0,06-0,08\end{array}$ & $\begin{array}{l}1,10-1,30 \\
0,08-0,09\end{array}$ & $\begin{array}{c}1-1,21 \\
\times \\
0,05-0,08\end{array}$ & $\begin{array}{l}1,29 \\
\times \\
0,06\end{array}$ & $\begin{array}{l}1,25 \\
\times \\
0,1\end{array}$ & $\longrightarrow$ & $1,4-1,5$ & - \\
\hline Bulbo & $\begin{array}{l}0,27-0,28 \\
0,20-0,26\end{array}$ & $\begin{array}{l}0,19-0,31 \\
0,19-0,30\end{array}$ & $\begin{array}{l}0,23-0,30 \\
0,23-0,26\end{array}$ & $\begin{array}{c}0,30 \\
\times \\
0,28\end{array}$ & 0,25 & - & $0,24-0,28$ & - \\
\hline Ovos & - & $\begin{array}{l}0,060-0,071 \\
0,041 \stackrel{x}{-0,045}\end{array}$ & & $\begin{array}{l}0,061-0,075 \\
0,041 \stackrel{\times}{-0,045}\end{array}$ & - & $\begin{array}{l}0,071 \\
\times \\
0,042\end{array}$ & - & $\begin{array}{l}0,078 \\
\times \\
0,043\end{array}$ \\
\hline Espículos & $0,81-0,98$ & $\longrightarrow$ & $0,76-1,1$ & - & $\begin{array}{c}1-1,1 \\
\times \\
0,1\end{array}$ & - & 0,75 & - \\
\hline Ventosa & $\begin{array}{l}0,11-0,14 \\
0,13-0,14\end{array}$ & - & $\begin{array}{l}0,11-0,15 \\
0,13-0,14\end{array}$ & $\longrightarrow$ & $0,13-0,15$ & - & - & . \\
\hline Ânus & $0,04-0,08$ & $0,27-0,30$ & $0,04-0,09$ & $0,30-0,32$ & 0,35 & - & - & $\longrightarrow$ \\
\hline $\begin{array}{l}\text { No de papilas } \\
\text { da cauda } \\
\text { do macho } \\
\end{array}$ & 10 Pares & $\longrightarrow$ & 10 Pares & - & 8 Pares & - & 10 Pares & - \\
\hline Hospedeiro & Tropidurus & rquatus (Wied) & Tro & urus & Tro & & Camal & erde \\
\hline Habitat & Intestir & grosso & Intestir & grosso & Intestir & osso & Intesti & osso \\
\hline Froveniência & Fortalez & - Ceará & São João - & Mato Grosso & São João - & - Grosso & $\begin{array}{l}\text { Angra } \\
\text { Estado do }\end{array}$ & $\begin{array}{l}\text { Reis } \\
\text { le Janeiro }\end{array}$ \\
\hline
\end{tabular}




\section{SUMMARY}

Contribution to the Knowledge of Strongyluris oscari Travassos, 1923

(Nematode, Subuluroidea).

The authors revise Strongyluris osca $r i$ Travassos, 1923, after studying the types and material from Fortaleza, Ceará State, rectify the original description and propose Strongyluris sai Travassos, 1926 as its synonym.

\section{REFERÊNCIAS BIBLIOGRÁFICAS}

ALHO, C. J. R., 1969, Oxyurata de lagar. tos do Planalto Central. Sobre o gênero Strongyluris Mueller, 1894 com descrição de duas espécies novas (Nematoda, Subuluroidea). Rev. Brasil. Biol., 29 (1) : 65-74, 12 figs.
ALHO, C. J. R., 1970, Frequency of infestation by Strongyluris treitasi Alho, 1969 in Tropidurus torquatus (Wied). Rev. Brasil. Biol., 30 (4): 539-542, 1 fig.

PEREIRA, C., 1935, Os Oxyurata parasitos de Lacertilia do Nordeste Brasi. leiro. Arch. Inst. Biol., 6 (1) : 5-27, 48 figs.

TRAVASSOS, L., 1923, Informações sobre a fauna helmintológica de Mato Grosso. Folba Méd., 4 (8) : 58.

TRAVASSOS, L., 1926, Sobre mais uma espécie do gênero "Strongyluris" Mueller, 1894, encontrada no Brasil. Ann. Fac. Méd. São Paulo, 1 : 83-84, 5 figs.

YAMAGUTI, S., 1961, Systema Helminthum. The nematodes of vertebrates. Pt. I e II, 1261 pp., 909 figs. Interscience Publishers, Inc. ed. New York.

YORKE, W., \& MAPLESTONE, P. A., 1926, The Nematodes Parasites of Vertebrates, VII + 536 pp., 307 figs., J. \& A. Churchill ed., London. 